\title{
PHOTO-REACTION OF IONIC BONDING PHOTOSENSITIVE POLYIMIDE
}

\author{
MASAO TOMIKAWA, MASAYA ASANO, GENTARO OHBAYASHI, HIROO HIRAMOTO \\ YOUTARO MORISHIMA* and MIKIHARU KAMACHI * \\ Eletronic and Imaging Material Research Laboratories, \\ Toray Industries, Inc. \\ Otsu, Shiga 520, Japan \\ *Faculty of Science, University of Osaka, \\ Toyonaka, Osaka 560, Japan
}

\begin{abstract}
Photoreaction of Ionic bonding photosensitive polyimide "Photoneece" was investigated by VIS spectroscopy, fluorescence spectroscopy, ESR spectroscopy, flash photolysis and quantitative analysis of chloroform extracts from prebaked film.

Main components of the photosensitive polyimide are poly(amic acid), tertiary amine having an acryloyl or methacryloyl group and a sensitizer. Surprisingly the acryloyl or methacryloyl group of the amine does not react by photo-irradiation. But the amine extracted by chloroform from the photosensitive polyimide film is reduced to one-half of its initial value after photoirradiation.

Polymer itself is excited by photo-irradiation to be an excited singlet state. After the intersystem crossing to an excited triplet state, an anion radical of pyromerittic diamide moiety in a polymer chain is generated. The formation of the anion radical is very slow (rate constant: $10^{5}(1 / \mathrm{sec}$ ) order). The anion radical is very stable (ca $\left.>10^{4} \mathrm{sec}\right)$.

An interaction of the anion radical and the tertiary amine seems to play an important role of the photosensitive polyimide.
\end{abstract}

\section{Introduction}

Ionic bonding photosensitive polyimide "Photoneece" ifs $^{2}$ developed first in the world by Toray of its own technology ${ }^{1}$.
The photosensitive polyimide has been applied to interdielectrics of semiconductors, multilayer circuit boards for computers and multichip-modules and protective coatings of semiconductors and line-sensors. Main components of this photosensitive polyimide are poly(amic acid) (polyimide precursor), tertiary amine having an acryloyl or methacryloyl group and a sensitizer. An ionic bonding is formed between a carboxylic group of poly(amic acid) 
and the tertiary amine.

It seemed that the double bond of the tertiary amine would polymerize by photo-irradiation. The polymerization would make a poly (amic acid) insoluble.

However it is found that most of triplet sensitizers, which show good effect for crosslinking formation of an acryloyl monomer, do not work. Some kinds of aromatic amines work effectively as a sensitizer. These facts suggest that the photoreaction of the photosensitive polyimide is different from a well-known photo-reaction. Clarifying the photo-reaction could be useful for developing a new photosensitive polyimide.

\section{Method}

\section{Sample preparation}

Poly (amic acid) (PAA)

$20 \mathrm{~g}$ of $4,4^{\circ}$-diaminodiphenylether(DDE) (0.1mole) was

dissolved in $160 \mathrm{ml}$ of $\mathrm{N}$-methyl-2-pyrollidone (NMP). $21 \mathrm{~g}$ of pyromerritic dianhydride (PMDA) (0.096mole) was added in this solution and stirred vigorousiy at room temperature under nitrogen for 1 hour and then at $50 \mathrm{C}$ for 3 hours.

\section{Photosensitive polyimide(PSP)}

$15.3 \mathrm{~g}$ of $\mathrm{N}, \mathrm{N}$-dimethylamino-ethyl-methacrylate (DMM) (equivalent to carboxylic groups of PAA) and $0.8 \mathrm{~g}$ of $\mathrm{N}$-phenyldiethanolamine (NDA) as a sensitizer dissolved in $10 \mathrm{~g}$ of NMP were added into the $100 \mathrm{~g}$ of poly(amic acid) solution. The solution was stirred for 2hours.

\section{Preparation of sample film}

Each solution of PAA, PSP, PAA with DMM (PAD) and PAA with NDA (PAN) solution was coated on a glass substrate by a spin coating method. Then coated samples were baked for 60min. at $80 \mathrm{C}$ in a convection oven under nitrogen.

\section{Interaction between poly(amic acid), DMM and NDA}

Fluorescent measurement was carried out with the Hitachi F4010 fluorescent spectrometer. (Excitation: 497nm, Monitor: 530$600 \mathrm{~nm}$ )

Interaction between the PAA and the DMM was investigated by quantitative analysis of the DMM in chloroform extracts from a PSP film and in DMF solution of the film after the extraction by use of the Shimadzu GC-8A gas chromatograph with a CBP-15 (25m) as a column. (Temperature range: from $100 \mathrm{C}$ through $240 \mathrm{C}$, Rate:16C/min., Detec :or:TCD, Detector current :100mA)

Change of DMM after photo-irradiation was analyzed by C-13 NMR spectroscopy using the JEOL FX-100 NMR spectrometer. (solvent DMSO-d6, $50 \mathrm{C}$ ) 
Determination of polymer radical

A radical was detected by ESR spectroscopy with the JEOL RE2-X ESR spectrometer with photo-irradiation. (Standard conditions: Magnetic field: $320-340 \mathrm{mT}$, Modulation: $100 \mathrm{kHz}$, $0.5 \mathrm{mT}$, Temperature -5 to $-150 \mathrm{C}$, Light source: $1000 \mathrm{~W}$ high pressure Hg lamp with a UV-39 filter)

Sample film was placed into a quartz tube sealed in vacuo.

An anion radical of pyromerittic diamide moiety was observed by VIS spectroscopy using the Shimadzu UV-260 spectrometer. The anion radical has an absorption maximum of $725 \mathrm{~nm}$.

Determination of reaction path

Reaction path was investigated by flash photolysis. Absorption spectra of a polymer films were obtained with an excitation of a 355nm laser pulse (3rd harmonic of a YAG laser) in the air. Sampling interval of these measurements was 0.1 microsec. or 20 microsec. A transient spectrum of excited states was obtained.

\section{Results}

Interaction between poly (amic acid), DMM and NDA

As shown in Fig.1, fluorescent intensity increases after photo-irradiation in PAN and PSP films. The intensity change of the PAN film is larger than that of the PSP film. The intensity of PAA and PAD films do not change significantly.

DMM contents in the chloroform extracts are reduced one-half of its initial value after photo-irradiation (Fig.2).

A reaction of DMM's double bonds was not observed by a C-13 NMR measurement shown in Fig.3.

\section{Determination of radical}

A radical is observed even in the PAA film by an ESR measurement with photo-irradiation ( $f i g .4)$. Signal intensity of the PAD, the PAN and the PAD films is larger than that of the PAA film. $G$ values are same among these 4 radicals. In the ESR spectra except for the PAA film, small signal with 4 lines having splitting constant $2.28 \mathrm{mT}$ are observed.

Wide band measurement $(80-580 \mathrm{mT})$ is carried out to investigate existence of a triplet radical pair. As shown in Fig.5, a signal is observed in a lower field (about 165mT, $g=4$ ).

\section{Determination of reaction path}

Results of flash photolysis are shown in Fig.6. Fluorescence is observed at an initial stage (about $0.05 \mathrm{microsec}$. after photoirradiation). After the fluorescence disappears, an absorption at $725 \mathrm{~nm}$ appears at about $6 \mathrm{microsec}$, after photo-irradiation. The absorption is very stable, still 6000micrsec. the peak height exists constantly.

The spectrum of VIS-spectroscopy after photo-irradiation (fig.7) is similar to the absorption spectrum of flash 
(a)

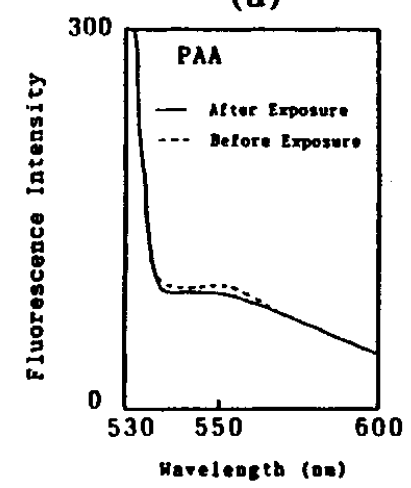

(b)

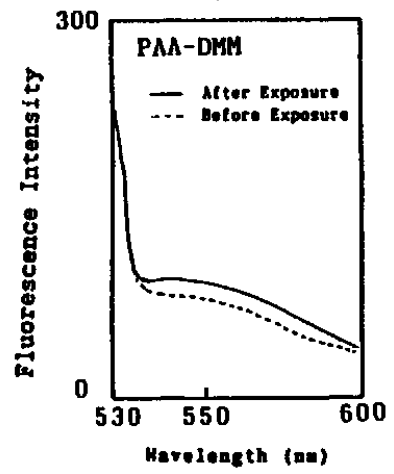

(c)

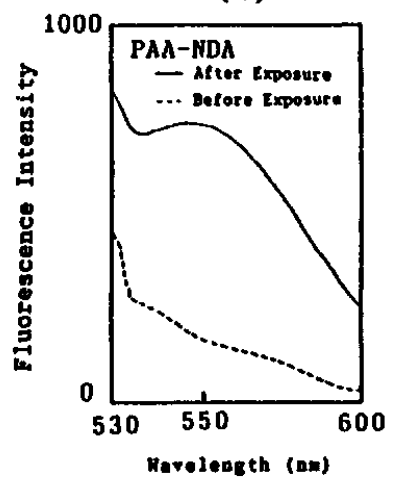

(d)

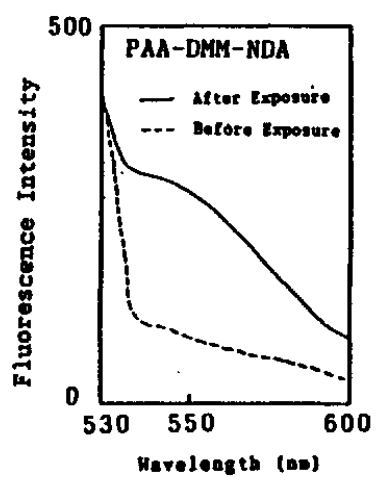

Fig.1 Fluorescence spectra of PAA(a), PAA-DMM(b), PAA-NDA (c) and PAA-DMM-NDA (d)

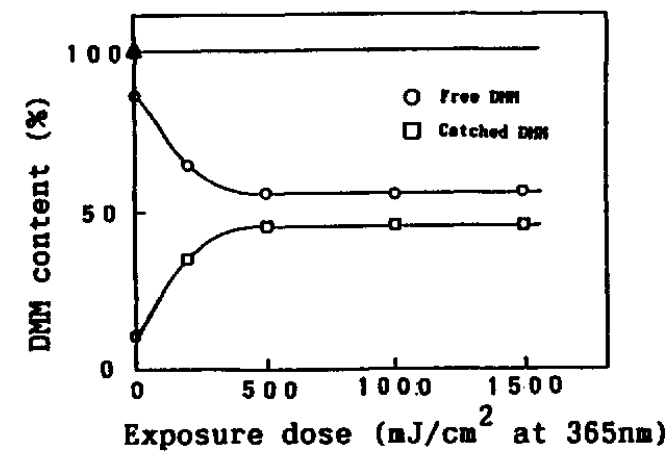

Fig.2 Interaction between PAA

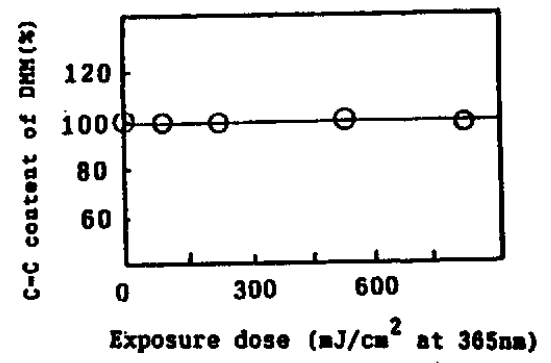

Fig. 3 Reaction of double bond of DMM with a photo-irradation

and DMM with a photo-irradiation

(a)

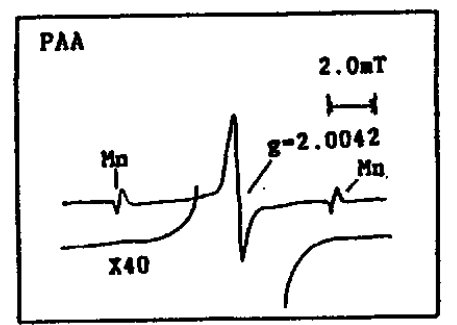

Magnetic Field

(c)

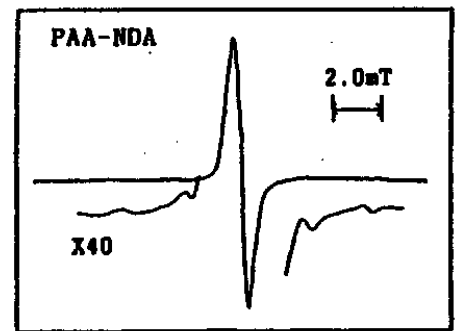

Magnetic Field (b)

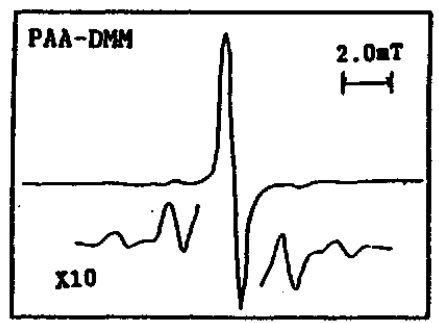

Magnetic Field

(d)

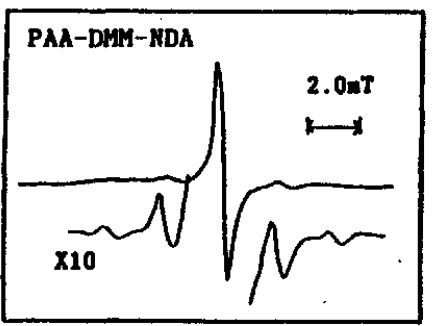

Magnet1c Fleld

Fig.4 ESR spectra of PAA(a), PAA-DMM(b), PAA-NDA(c) and PAA-DMM-NDA(d) 
photolysis after 6microsec. The absorption disappears about 150min. after photo-irradiation.

\section{Discussion}

\section{Initial reaction}

The photosensitive polyimide shows the fluorescence immediately after photo-irradiation. 4 film samples mentioned above are different in the change of the fluorescent intensity caused by photo-irradiation. In case of the PAN film, fluorescent intensity increases significantly. Fluorescent intensity of the PSP film increases but the change of intensity of it is less than that of the PAN film. In case of the PAD film, fluorescent intensity changes a little.

These results show that the NDA assists the PAA in photoexciting, while the DMM quenches the fluorescence.

\section{Information of excited state}

As mentioned above, the small splitting signals are observed in all films except for the PAA. It is well-known that a radical of methacryloyl group shows 5 lines. But this splitting signal is observed in the PAN film. The PAN film is composed of PAA and NDA which do not have methacryloyl group. The signal is not derived from the methacryloyl group. The signals are symmetry at a point of convergence. This phenomenon is observed when a triplet radical pair exists in a system. The result of wide band measurement supports existence of triplet radical pair.

The excited singlet state changes to the excited triplet state in this system at an initial stage by an intersystem crossing.

\section{Radical structure}

The PSP film shows a broad absorption spectrum of which peak is about $725 \mathrm{~nm}$ by the results of flash photolysis.

Morishima et $\mathrm{al}^{2}$ ) and Mataga et al ${ }^{3)}$ reported that an anion radical of pyromerittic dianhydride shows an absorption at $666 \mathrm{~nm}$ by a flash photolysis.

The absorption spectra of the PSP is similar to that of the anion radical of pyromerittic dianhydride. Absorption maximum of the PSP is about 60nm longer than that of the anion radical of pyromerittic dianhydride.

The absorption at $725 \mathrm{~nm}$ seems to be assigned to an anion radical of pyromerittic diamide molety in the polymer chain. The pyromerritic diamide moiety has a long conjugation prolonged to a benzene ring by its amide bond. The conjugation causes the absorption peak to shift to longer wave lengths.

\section{Formation of an anion radical of pyromerritic diamide}

The anion radical of pyromerittic diamide moiety appears at about 6microsec. after photo-irradiation. Formation of the radical is rather slowly. 


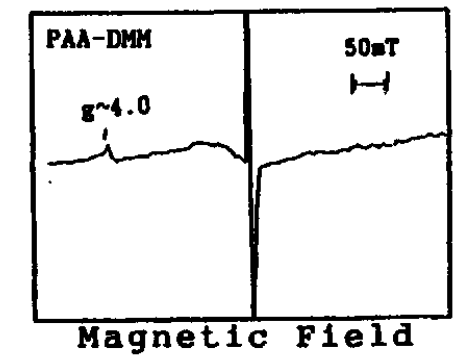

Fig. 5 Wide range ESR spuctum of PAA-DMM
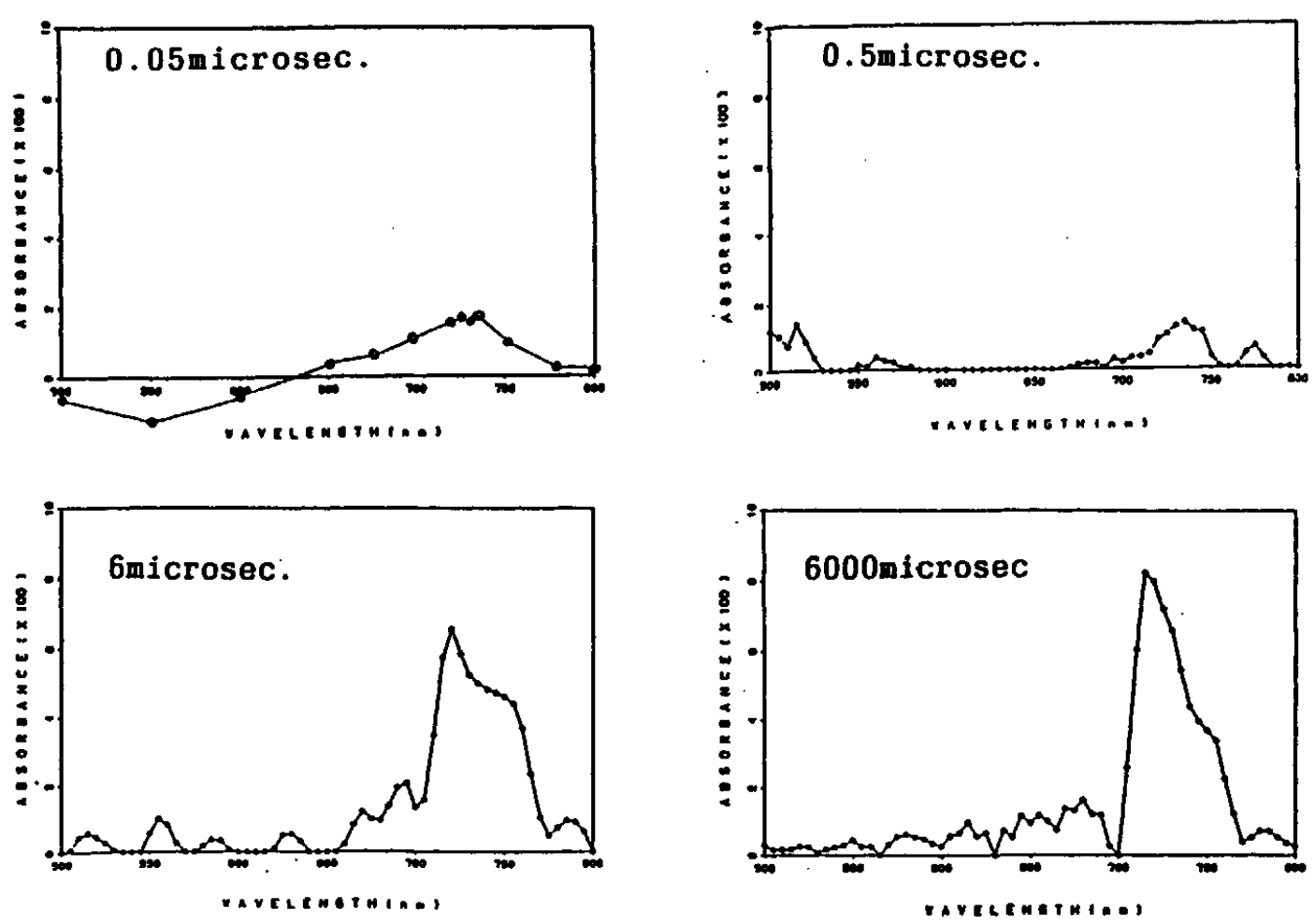

Fig.6 Transient spectra of PAA-DMM-NDA

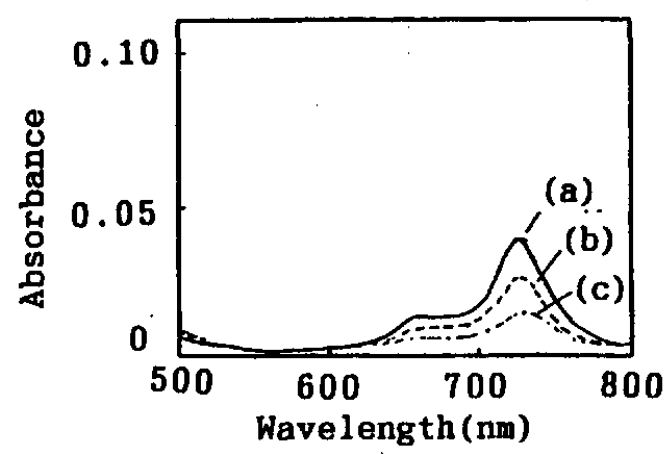

Fig. 7 VIS-spectroscopy of PAA-DMM-NDA

(a) 20min. after irradiation

(b) $45 \mathrm{~min}$. after irradiation

(c) $60 \mathrm{~min}$. after irradiation 
The radical is very stable even in the air in the PSP film. on the contrary, any anion radical is not observed in the PAN film which have not DMM. It seems that there is some kind of interaction between the anion radical and DMM. Such effect is caused from electron attracting nature of the DMM's methacryloyl group. Existence of the interaction is supported by the fact that the DMM extracted by chloroform from the PSP film is reduced after photo-irradiation.

\section{Reaction path} is assumed.

From the results we discussed above, following reaction path

At first the PAA itself is excited to the singlet state by photo-irradiation. This excitation is enhanced with an aromatic amine such as NDA. The Excited singlet state changes to the excited triplet state by the intersystem crosing. The rate constant of the intersystem crossing is estimated to be $10^{8}$ $(1 / \mathrm{sec})$ order from the results of flash photolysis.

Formation of the anion radical of pyromerittic diamide molety is rather slowly (rate constant: $10^{5}$ (1/sec.) order). This anion radical is very stable even in the air (life time: $10^{4} \mathrm{sec}$. order) because of the electron attracting nature of the DMM's methacryloyl group. This interaction seems to make a weak cross linking between polymer chains.

A postulated reaction path is shown in Fig.8.

\section{Conclusion}

Photoreaction of lonic bonding photosensitive polyimide was investigated.

An anion radical of pyromerittic diamide moiety in the polymer chain is generated after photo-irradiation of the PSP film. A weak crossilnking formed by lonic bonding and interaction between the anion radical and a tertiary amine seems to make the PSP insoluble to the developer.

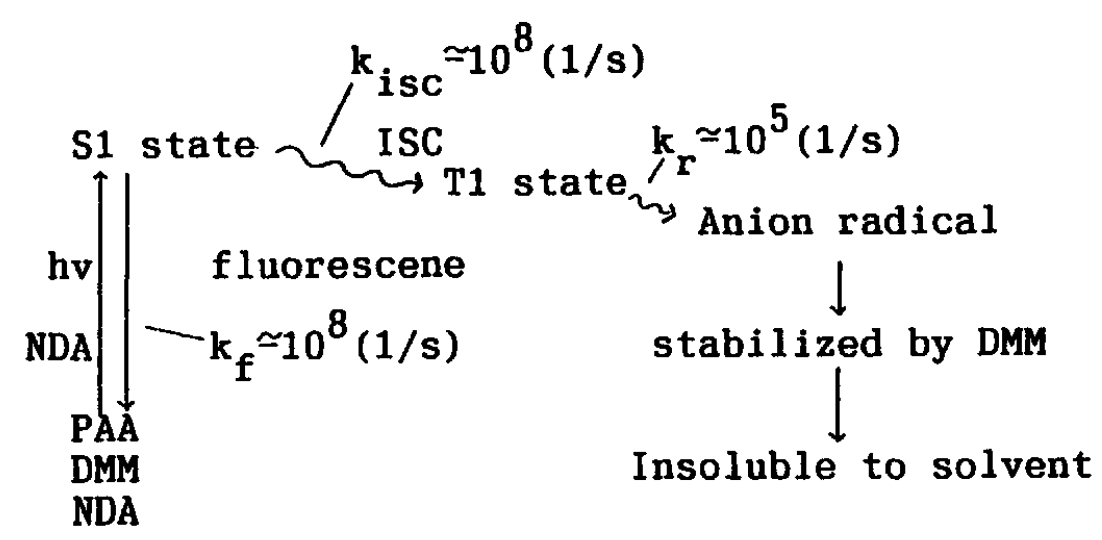

Fig. 8 Postluate reaction path of this work 


\section{Acknowledgment}

The Authors express great appreciation to Dr.Kajiwara (Faculty of Science, Univ. of Osaka) for measuring supports and useful suggestions.

\section{References}

1. N.Yoda, H.Hiramoto et al, J. Macromol. Sci. (Chem.) A21,1641 (1984)

H.H1 ramoto and M.Eguchi, JP.1520755

H.Hiramoto and M.Eguchi, USP.4243743

2. M.Sek1, Y.Tominaga, Y.Morishima and M. Kamachi, Symposium on photochemistry, 371, (1990)

3. M.Shimada, H.Masuhara and N.Mataga, Bull Chem. Soc. JPN. 46, 1903(1973) 